14

\title{
Квантовый нейристор: от теории к эксперименту
}

\author{
() С.Л. Григорьев
}

620142 Екатеринбург, Россия

e-mail: sir.grig2011@yandex.ru

\section{(Поступило в Редакцию 14 ноября 2016 г.)}

На основе квантово-оптической модели живого нейрона и современных достижений электроники и оптоэлектроники намечены пути теоретических и экспериментальных работ для проверки этой модели с целью создания нового элемента для нейросетевой информатики - квантового нейристора.

DOI: 10.21883/JTF.2017.07.44689.2096

Фон Нейман в середине прошлого века рассматривал нейросетевые системы как наиболее перспективные для создания устройств информатики. Однако развитие электроники и вычислительной техники пошло по пути создания более простых числовых устройств на бинарных пороговых элементах — триггерах. В 2003 г. за рубежом была предложена наноструктура [1] из пересекающихся проводников (рис.1), которая оказалось пригодной для создания многих нанопроцессоров, в том числе нейроподобных [2-6].

Согласно опубликованной отечественной модели живого нейрона [7], он работает аналогично лазеру с параллельной накачкой [8]: многие входящие нервные волокна возбуждают мембрану тела нейрона, содержащую бистабильные молекулы, мембрана генерирует выходной импульс, который распространяется в длинное волокно нейрона - аксон (рис. 2). Сигналы могут распространяться в волокне мембраны посредством перескоков на молекулярных группах липидов и полипептидов, как показано в работах А.С. Давыдова [9]. Следует отметить, что базовое когерентное состояние нейрона не создается входящими нервными волокнами. Оно создается фрелиховскими биоколебаниями, генерируемыми нейроном и любой другой живой клеткой за счет биохимических реакций [10]. Можно предположить, что нейрон совершает квантовое сложение двух нечетких множеств сигналов:

- поступающих от рецепторов или других нейронов,

- хранящихся в синапсах перед проведением сложения.

Профессор Эмилио Джудич [11] на основе квантовой теории поля оценил энергию этих колебаний величиной $0.3 k T$, что соответствует оптическим фононам согласно современным данным квантовой электроники [12] и диапазону частот от 50 до $300 \mathrm{~cm}^{-1}$ по данным физических методов исследования аминокислот и других сложных биомолекул [13]. Биоколебания характеризуются большой амплитудой (до $1 \mathrm{~nm}$ ) и ангармоничностью.

Согласно [7], потенциалу действия живых нейронов, известному в физиологии, соответствует пороговая мощность излучения накачки на входе в активный элемент искусственных квантовых нейристоров - многоканальных логических квантовых генераторов. Пороговый эффект для лазера с одноуровневой схемой возбуждения описывается формулой [14]:

$$
P=\left(V_{1} / V_{2}\right)\left(k_{1} / k_{2}\right)\left(\lambda_{1} / \lambda_{2}\right)(1-\exp (-k L))\left(P_{1}-P_{t h}\right),
$$

где $P$ - выходная мощность, $P_{1}-$ мощность накачки на входе в активный элемент, $P_{t h}-$ пороговая мощность излучения накачки на входе в активный элемент, $V_{1}, V_{2}$ - объем, который занимает излучение накачки и генерируемое излучение в активном элементе, $k_{1}, k_{2}-$ коэффициенты потерь излучения накачки и генерируемого излучения, $\lambda_{1}, \lambda_{2}$ - длины волн излучения накачки и генерируемого излучения.

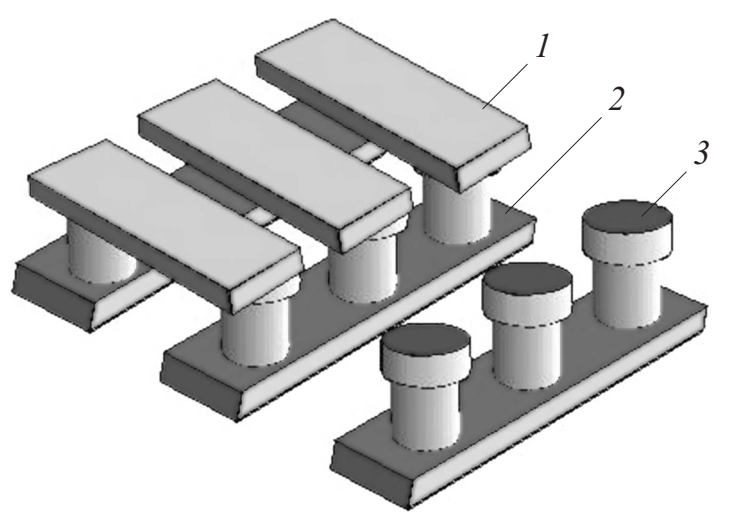

Рис. 1. Структура из пересекающихся нанопроводников: 1 верхний ряд проводников, 2 - нижний ряд проводников, $3-$ нелинейные электропроводящие элементы.

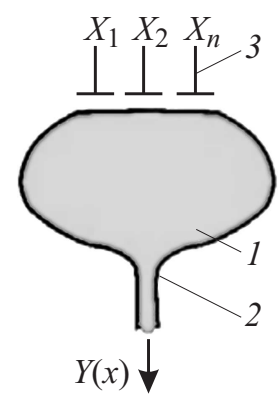

Рис. 2. Схема живого нейрона: мембрана тела нейрона (1), выходное волокно (аксон) и выходной сигнал (2), входы сигналов от нервных волокон с синапсами (3). 


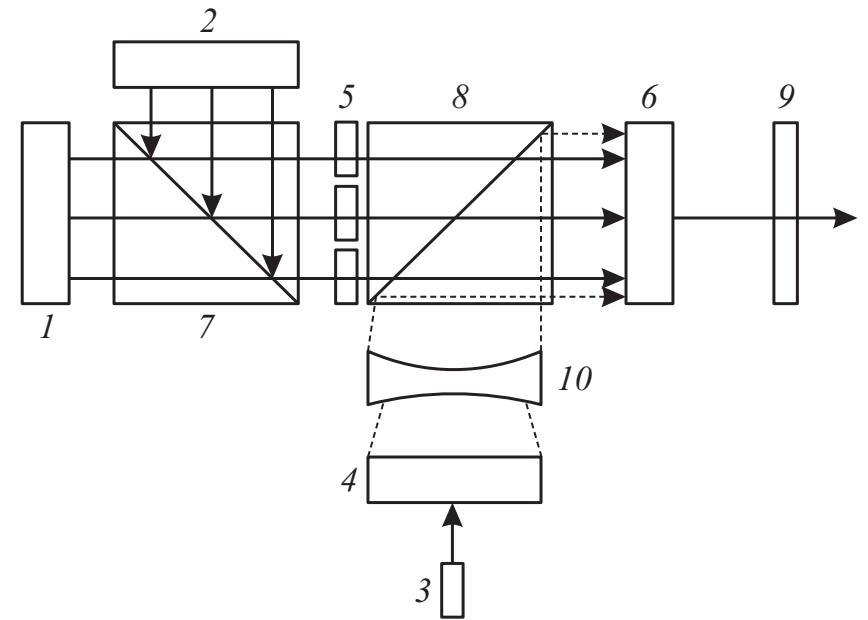

Рис. 3. Возможная схема экспериментальной установки, имитирующей работу нейрона: 1 - матрица InGaAs диодов, создающая распознаваемый образ; 2 - матрица InGaAs диодов, создающая эталонный образ; 3 - InGaAs диод накачки Er : YAG лазера базовой накачки основного логического лазера; 4 $\mathrm{Er}$ : YAG лазер базовой накачки, который может использоваться для накачки нескольких нейристоров и их когерентной синхронизации; 5 - матрица $\mathrm{Er}$ : YAG лазеров, осуществляющих нелинейное (пороговое) сложение двух входящих сигналов (элементов матриц 1 и 2); эти лазеры являются аналогами синапсов нейрона; 6 - основной логический лазер, аналог тела нейрона; 7,8 - поляризационные кубики - линейные сумматоры сигналов; 9 - выходное зеркало; 10 - линза.

Таким образом, у квантового нейристора есть аналогия с матричным векторным процессором, а наиболее полным аналогом является голографический координатор [15]. Структура с пересекающимися нанопроводниками (рис. 1) в принципе пригодна для технического моделирования квантового нейристора. На первом этапе это может быть устройство для работы с матрицами, состоящими из нефазированных электрических или оптических сигналов. Однако в настоящее время еще не накоплено достаточно опыта для создания таких структур с оптически активными элементами.

Тем не менее базовые принципы работы квантового нейристора могут быть проверены не только на наноструктурах, но и на лазерах любого типа, например на хорошо освоенных YAG-лазерах с параллельной диодной накачкой [8]. Диоды могут обеспечить базовую накачку излучателя, создать матрицы эталонного образа и распознаваемого образа. При теоретическом анализе принципов работы такого устройства не следует пренебрегать низколежащими (фононными) энергетическими уровнями логического лазера $[12,16]$, которые могут быть использованы для создания базовой когерентности аналогично фрелиховским биоколебаниям в живом нейроне. Конструкция прототипа квантового нейристора может быть рассчитана и теоретически обоснована. Примеры теоретических подходов и расчетов лазерных устройств, в том числе YAG-лазеров разного назначения, приведены в работах $[14,16]$.

В качестве примера экспериментальной установки рассмотрим возможную квантово-оптическую схему, показанную на рис. 3. В схеме (рис. 3) предлагается использовать кристаллы $\mathrm{Er}$ : YAG, энергетические уровни и переходы в которых показаны на рис. 4. Базовую накачку основного кристалла нейристора 6 ниже порогового уровня $P_{t h}$ предлагается производить от такого же лазера на основной волне излучения $2.9 \mu \mathrm{m}$. Это позволит от одного лазера базовой накачки 4 производить когерентную накачку нескольких параллельно работающих нейристоров. Для накачки этого лазера можно использовать InGaAs-диод с длиной волны $980 \mathrm{~nm} 3$. Сигнал от распознаваемого образа и эталонный сигнал из памяти должны складываться нелинейно, т.е. только при их совпадении. Это свойство синапсов известно из физиологии и теории нейронных сетей [17]. Данная проблема решается применением матрицы кристаллов Er: YAG 5, с накачкой матрицей InGaAs-диодов 1. Линейное сложение сигналов от матриц 1 и 2 производится в поляризационных кубиках 7,8 [8]. Размер матриц $n x m$ зависит от технологических возможностей и сложности распознаваемых образов. Современные YAG-микролазеры при выходной мощности менее $0.1 \mathrm{~mW}$ могут иметь размеры, измеряемые миллиметрами.

Может производиться не только сравнение образов (фигур), но и любой совокупности сигналов, необходимых для принятия решений в системах автоматики.

Более детально структура распознавания образов мозгом изучена и описана Нобелевскими лауреатами Д. Хьюбелом и Т. Визелом [18]. В частности, они обнаружили в мозге колоночные структуры нейронов,

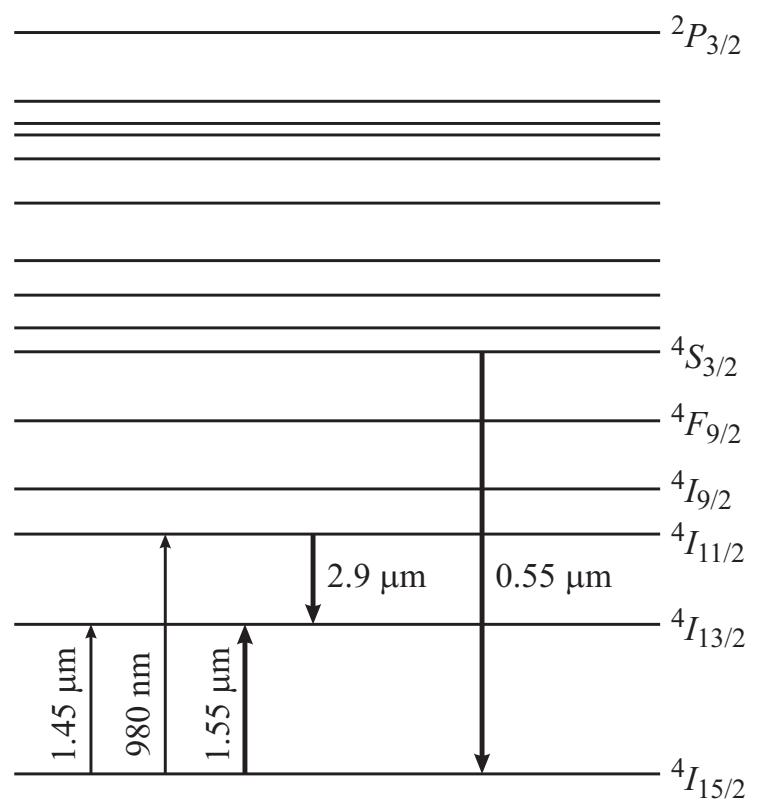

Рис. 4. Энергетические уровни и переходы в лазерном кристалле Er : YAG (по данным www.sctic.ru). 
ответственные за распознавание отдельных элементов воспринимаемых образов.

Создание квантового нейристора может быть полезно для более глубокого понимания работы мозга, развития нейросетевых компьютеров и создания искусственного интеллекта [19].

\section{Список литературы}

[1] Chen Y., Jung G.-Y., Ohlberg D.A.A., Li X., Stewart R.D., Jeppesen J.O., Nielsen K.A., Stoddart J.F. // Nanotechnology. 2003. 2003. Vol. 14. P. 462-468.

[2] Borghetti J., Li Z., Straznicky J., Li X., Ohlberg D.A.A., Wu W., Stewart D.R., Williams R.S. A hybrid nanomemristor/transistor logic circuit capable of selfprogramming. PNAS (Proc. Natl. Acad. Sci. USA). 2009. Vol. 106. N 6. P. 1699-1703.

[3] Green J.E., Choi J.W., Boukai A., Bunimovich Y., JohnstonHalperin E., Delonno E., Luo Y., Sheriff B.A., Xu K., Shin Y.S., Tseng H-R., Stoddart J.F., Heath J.R. // Nature. 2007. Vol. 445. P. 414.

[4] Jo S.H., Chang T., Ebong I., Bhadviya B.B., Mazumder P., Lu $W$. // Nano Lett. 2000. Vol. 10. P. 1297-1301.

[5] Kuzum D., Jeyasingh R.G.D., Lee B., Wong H-S.P.// Nano Lett. 2012. Vol. 12. P. 2179-2186.

[6] Yan H., Choe H.S., Nam SW., Hu Y., Das S., Klemic J.F., Ellenbogen J.C., Lieber C.M. // Nature. 2011. Vol. 470. P. 240-244.

[7] Григорьев С.Л. // ЖТФ. 2002. Т. 72. Вып. 2. С. 16-19.

[8] Кравцов Н.В. // Квантовая электроника. 2001. Т. 31. № 8. C. 661-677.

[9] Davidov A.S. // J. Theor. Biol. 1977. Vol. 66. P. 379.

[10] Frohlich H. Biological Coherence and Response to External Stimuli / Ed. by H, Frohlich. Springer-Verlag, 1988. P. 1-24.

[11] Del Giudice E., Doslia S., Milani M., Vitiello G. Biological Coherence and Response to External Stimuli. Ed. by H. Frohlich. Springer-Verlag, 1988. P. 49-64.

[12] Stroscio M.A., Dutta M. Phonons in Nanostructures. Cambrige Univ. Press, 2001.

[13] Trueblood K.H. Accurate Molecular structures. Their Determination and Importance / Ed. by A. Domenicano, I. Hargittai. Oxford Univ. Press, 1992; Gramaccioli C.M. ibid; Jeffrey G.A., ibid.

[14] Ивашко А.М., Кисель В.Э., Ясюкевич А.С., Кулешов Н.В. // Приборы и методы измерений. 2014. № 2. С. 17-20.

[15] Кухаркин E.C. Электрофизика информационных систем: Учеб. пособие для вузов. М.: Высш. школа, 2001.

[16] Петрушкин С.В., Самарцев В.В. Лазерное охлаждение твердых тел. М.: Физматлит, 2005.

[17] Тарков М.С. Нейрокомпьютерные системы. М.: ИнтернетУн-т инф. знаний БИНОМ, 2006.

[18] Hubel D. Eye, Brain, and Vision / www.hubel.med.harvard.edu

[19] Grigor'ev S.L. // Int. J. Contemporary Applied Sciences. 2016. N 9. P. $104-110$. 